\title{
Analysis of Pedological Traces in Forensic Practice and New Possibilities in This Field
}

\author{
Marek Kotrlý ${ }^{1,2}$ \\ 1. Institute of Criminalistics Prague (ICP), Czech Republic \\ 2. Charles University in Prague, Faculty of Science, Praha, Czech Republic.
}

An essential feature differs forensic labs from other scientific facilities, while all other labs are for some time focused on investigation of materials of one type, to a forensic lab can be submitted practically any material related to a crime or crime scene. This is in practise any material of nature origin or any material produced by a human being. A forensic lab should be able to determine this material at least generally and classify it. This wide range also refers to pedological traces that may include a complex material and therefore forensic analysis of soils belongs to the most comprehensive investigations in a forensic field.

Analysis of pedological phases that adhered to clothing, footwear, vehicles, or other objects is relatively frequently required in forensic practice to confirm the place of origin of contamination. These are basically two types of tasks. This entails either standard comparisons, when the questioned soil phases are compared with collected reference samples to confirm the crime scene, vehicle route, etc. Alternatively, forecasting of an unknown location is required where contamination by soil may have occurred. These are in both cases complex analyses, in which the organic component of the sample is studied separately, possibly anthropogenic contamination and mineral material itself.

Basic techniques are optical light microscopy in transmitted and reflected light, polarization, fluorescence, next are utilised SEM/EDS (WDS), XRF and FTIR. For the clay fraction, but also for other phases, the x-ray diffraction is imperative with applying conventional powder diffraction in transmission and reflected modes, scanning in a rotating capillary, and also micro x-ray diffraction, which allows to obtain a relevant structural record from the diameter around $0,1 \mathrm{~mm}$. Technique of cathode luminescence (CL) capable of distinguishing material based on its genesis, thus usually also according to a different place of occurrence, were introduced to differentiate mineralogical phases of practically identical chemical composition, optical characteristics, structure and similar inclusions. Biological material frequently present in the traces is analysed separately, plant and animal relicts are analyzed, including microscopic shells and relics (e.g. group Diatoms, etc.). Anthropogenic material (glass fragments, slag, construction materials, etc.) is analyzed separately by other techniques and may increase the probability measure of a match (hit) between traces and reference samples. For forecasting of unknown locations, where the contamination by soil may have occurred, systems of geographic information (GIS) are used, in which data are connected with detailed geological maps, both uncovered and covered, and with pedological maps. A detailed configuration of the field in a respective site is crucial for the assessment of all circumstances related to the detected trace, likewise employing 3D terrain models with a detailed topographic base and 3D models using orthophoto maps.

The complex of methodologies is based on mineralogical and physical-chemical methods better embracing particularities of soil composition than some applied procedures arising from purely chemical base. Nevertheless, the introduced system at the same time is not omitting even biological and anthropogenic materials that usually represent a very important comparative material. 
Genetic methods that could compare both information from plant fragments and soil microorganisms are a big potential into the future.

Most of the analytical methods described yield quantitative data for data comparison. One of the possibilities bringing to soil analysis quantitative data that can be used e.g. for statistical comparison of traces and reference samples among each other are systems of automatic analysis of mineral grains (based on SEM/EDS). These systems have been available at the market for several years, nevertheless, to date samples of mineral grains recovered from soil traces have been prepared into casting compounds and then have been cut into polished section forms. Only then were samples analysed in systems of automatic mineralogical analysis. Our laboratory for the first time carried out in cooperation with the producer a number of detailed experiments with analysis of samples prepared directly from mineral grains of a soil trace, fixed at an adhesive stub with carbon target in diameter of $30 \mathrm{~mm}$. Experiments were at first conducted with model samples prepared from known mineral phases and then with samples from common soils. Several series of random samples were taken in a final stage from different places and the ability to determine differences among individual sampling points was tested. It was found that the differences are traceable also among sampling sites that were at a distance less than $20 \mathrm{~m}$. This technology, of course, is not a panacea, but appropriately compliments a complex analysis of soil phases.

The use of new methods considerably extends possibilities of typical microscopic procedures in a forensic field and allows to obtain the needed quantitative data in a forensic analysis of pedological phases, differentiation of analogous mineral phases, or the option of analysis of the organic phase directly in SEM chamber. Good experience was obtained with some systems of automatic analysis of mineral grains (based on SEM/EDS), analysing several hundred up to thousands of mineral grains, carrying out their automatic classification according to chemical composition and subsequently comparing samples by statistical methods. The technology described suitably supplements the complex analysis of soil phases. [4]

\section{References:}

[1] Kotrlý M., Turková I.: Proc. SPIE 9073, 2014, SPIE, 90730U-1 - 10, Vol. 9073, 2014.

[2] Kotrlý M., Turková I.: Proc. SPIE 9486, 2015, SPIE 948614-1 - 11, Vol. 9486, 2015.

[3] Kotrlý M.: Microsc. Microanal. 21 (Suppl 3), pp. 1365 - 1366, 2015.

[4] Acknowledgements - microanalytical methods at ICP were supported by projects: VD20062008B10, VD20072010B15, VG20102015065, VF20112015016, VF20122015027, VI20152020035. 